\title{
Adolescent/Young Adult (AYA) Hematology/Oncology Patient and Parent Attitudes toward AYA COVID-19 Vaccination
}

\author{
Sara L. Hardman ${ }^{1}$, Mahvish Q. Rahim², Meagan E. Miller ${ }^{1}$, Scott L. Coven ${ }^{2}$, Seethal A. Jacob², \\ Gregory D. Zimet ${ }^{2}$, Carolyn G. Meagher ${ }^{2}$, Mary A. Ott ${ }^{2}$ \\ ${ }^{1}$ Indiana University School of Medicine; ${ }^{2}$ Indiana University School of Medicine, Department of \\ Pediatrics
}

\section{Background:}

Adolescent/young adult (AYA) patients with hematologic and oncologic conditions are at increased risk for complications of COVID-19 and thus are important targets for vaccine outreach. AYA patients are transitioning from relying upon parental vaccine decision-making to independently making their own decisions. AYA with sickle cell disease (SCD) are of particular concern because a high proportion are African American and experience structural racism in addition to their illness. Further, AYA patients with chronic conditions may consider their past and present illness in their decision-making process.

\section{Methods:}

As part of a larger IRB-approved study, we recruited vaccine decision-makers for AYA patients aged 9-21 years attending SCD and oncology survivor clinics, including AYA patients 18-21 years old and parents of AYA patients 9-21 years old. After informed consent, participants completed a short demographic survey and a semi-structured interview regarding their vaccine decision-making process. Questions about the COVID-19 vaccine were incorporated given the ongoing pandemic.

\section{Results:}

Forty-nine parents and 21 AYA patients were recruited. The primary barriers reported regarding vaccination were concerns about its short-term side effects (57\% AYAs; $37 \%$ parents) and potential to have unknown, long-term effects (10\% AYAs; $14 \%$ parents). There were also concerns voiced about how rapidly the vaccine was developed (14\% AYAs; $27 \%$ parents) and misconceptions about the vaccine (19\% AYAs; $10 \%$ parents). Parents and AYA patients described the benefits of vaccination as lowering personal risk (62\% AYAs; $35 \%$ parents) and several also mentioned the community benefits of preventing the spread of COVID-19 (19\% AYAs; $8 \%$ parents) and a possible return to "normal" (14\% AYAs; $10 \%$ parents).

\section{Potential Impact:}

The data from this study will further the understanding of how parents and young adults with chronic hematologic and oncologic conditions make decisions about COVID-19 vaccination, a vital tool for protecting medically and socially vulnerable populations during the COVID-19 pandemic. 